EPJ Web of Conferences 59, 07006 (2013)

DOI: $10.1051 /$ epjconf/20135907006

(C) Owned by the authors, published by EDP Sciences, 2013

\title{
Improvement of the spatial and temporal contrast of short-pulse KrF laser beams
}

\author{
S. Szatmári ${ }^{1}$, R. Dajka ${ }^{1}$, A. Barna ${ }^{1,2}$ and I.B. Földes ${ }^{2, a}$ \\ ${ }^{1}$ University of Szeged, Department of Experimental Physics, Szeged, Hungary \\ ${ }^{2}$ Wigner Research Centre for Nuclear Physics, Association EURATOM HAS, Budapest, \\ Hungary
}

\begin{abstract}
Important figure of merit of high-intensity laser systems is the temporal and spatial quality of their pulses. Spatial filtering is a well known technique to improve the spatial quality by modulating the spatial components at the Fourier-plane, using a pinhole of appropriate size or recently by a nonlinear process. Modulation of the beam in the Fourier-plane allows however a simultaneous spatial and temporal filtering. By the use of a "conjugate" pinhole arrangement before and after the nonlinear spatial selector, intensity dependent transmission is obtained: the low intensity part is efficiently suppressed. Numerical calculations predict practical operation for both amplitude and phase modulation at the Fourier-plane. In the preferred latter case the experimental observations are in good agreement with the theory, demonstrating $>40 \%$ throughput.
\end{abstract}

\section{INTRODUCTION}

Major figure of merit of high-intensity laser systems is how efficiently they can temporally and spatially concentrate the energy carried by the pulse. Short-wavelength lasers have better theoretical capability for such temporal and spatial compression, which, however, can only be utilized, if their optimum beam quality is maintained [1]. Short-pulse excimer laser systems are generally dual-wavelength lasers, where the generation and the final amplification of the pulse are carried out at two different wavelengths necessitating frequency doubling or tripling before UV amplification [2].

Using a novel way for frequency doubling - called active spatial filtering [3] - not only the inherent temporal cleaning of the intensity dependent nonlinearity occurs, but also efficient spatial filtering can be realized. This leads to a double wavelength system where the temporal and spatial contrast are reset in the "middle" of the system, resulting in output pulses of excellent spatial and temporal quality for medium output power. For larger output energy, however, the rapidly growing amplified spontaneous emission (ASE) in the UV amplifiers deteriorates the temporal contrast.

Recent development of Ti:Sapphire lasers allowed to enter the attosecond regime and to give the highest peak powers. It is a single-wavelength laser where the pulse cleaning technique based on nonlinear frequency conversion cannot be used. Due to the long wavelength, it is not the ASE, but the temporal background - associated with the Chirped Pulse Amplification (CPA) scheme - which mainly deteriorates the temporal contrast. For these reasons intense ultrashort laser pulses generally suffer from prepulses which may originate either from the ASE of the UV amplifier chain or from the pedestals due to the imperfect pulse compression. Prepulses are detrimental for high intensity laser-material interactions, as the longer prepulse may generate a preplasma. In such case, the main laser pulse interacts

\footnotetext{
ae-mail: foldes.istvan@wigner.mta.hu
}

This is an Open Access article distributed under the terms of the Creative Commons Attribution License 2.0, which permits unrestricted use, distribution, and reproduction in any medium, provided the original work is properly cited. 


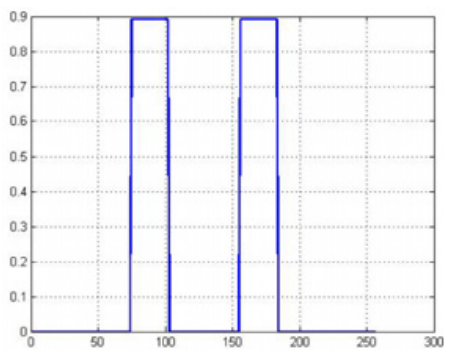

a)

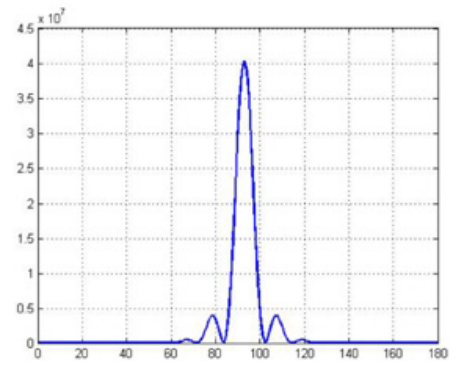

b)

Figure 1. Cross section of an annular beam (a) and its distribution in the focal plane (b).

not with the solid target but with a preformed plasma. It was recently shown [4] that even nonionizing infrared prepulses of $10^{8} \mathrm{~W} / \mathrm{cm}^{2}$ intensities can change laser matter interactions considerably. In the $\mathrm{UV}$, free ions generated by photoionization were detected and reported to modify the interaction below $10^{7} \mathrm{~W} / \mathrm{cm}^{2}$ intensity [5]. For the highest intensities temporal contrast beyond $10^{10}$ is needed.

One of the most efficient methods to remove prepulses is based on the self-induced plasma shuttering or plasma mirror technique [6], which was successfully demonstrated for short-pulse KrF laser systems, too [7]. If the intensity of the laser pulse falling onto a transparent solid material is chosen so that only the leading edge of the main pulse is above the plasma formation threshold, prepulses or the pedestal of lower intensity will be suppressed, improving the contrast up to several orders of magnitude. The achievable contrast improvement with the use of plasma mirrors is limited by the limited ratio of the high and low intensity reflectivity. Therefore significant contrast improvement can only be achieved by subsequent use of mirrors, which however limits the overall throughput of the system. Disadvantages of plasma mirrors are that they are positioned into a beam of finite size, where the optical quality of the plasma front influences the phase front of the beam and that a fresh target area is needed for each shot.

For these reasons we are looking for a pulse-cleaning technique, which is generally applicable both for single and dual wavelength lasers, and does not suffer from the shortcomings of the standard plasma mirror method.

\section{DESCRIPTION OF THE METHOD}

The basic idea behind the suggested method is similar to that of the active spatial filtering: an intensity dependent modulation introduced in the Fourier-plane leads to directional modulation. For such purpose an annular input beam (Figure 1a) seems ideal having an "Airy-like" intensity distribution in the Fourierplane (Figure 1b) whose nonlinear modulation has already been proven to generate a "Gaussian-like" output beam [3]. Then, in a newly suggested arrangement, the plasma mirror is situated in the center of a confocal telescope surrounded by a conjugated beam-block filter pair similar to the arrangement in Fig. 3. As long as no amplitude (and/or phase) modulation occurs in the focal plane, this arrangement has no transmission, allowing full exclusion of eventual prepulses. However, for the intense main pulse, where the plasma-mirror causes an amplitude modulation in the focal plane, finite transmission occurs.

Figure $2 \mathrm{a}$ shows the calculated distribution when the intensity of the higher (than $0^{\text {th }}$ ) orders are suppressed by a factor of 25 (e. g. by a plasma mirror). In this case a fraction of the energy is diffracted to the central part (to the hole of output filter) resulting in finite transmission, and significant improvement of the contrast. The overall transmission is expected to be poor due to the diffraction losses and the limited plasma reflectivity $[5,6]$.

Much better results are obtained and simultaneous temporal and spatial filtering occurs, when phase modulation is introduced in the focal plane instead of amplitude modulation. Figure $2 \mathrm{~b}$ shows the results 


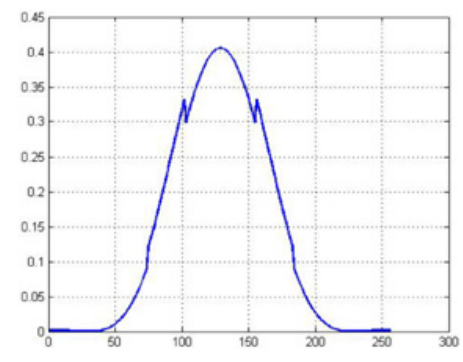

a)

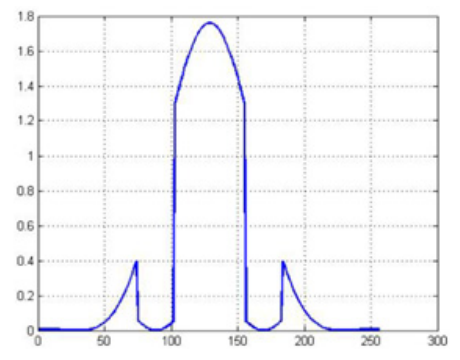

b)

Figure 2. The output beam after the confocal telescope for $25 \times$ amplitude modulation (a) and for a phase modulation with $\lambda / 2$ (b).

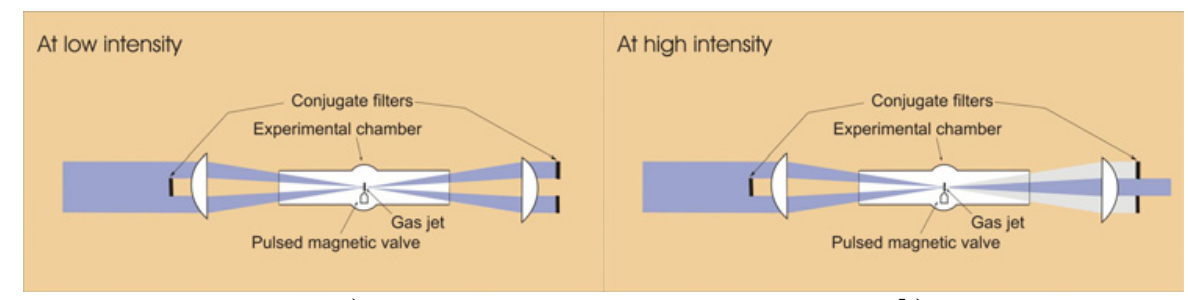

a)

b)

Figure 3. Experimental arrangement for spatial filtering with phase modulation. In case of low intensities there is no phase shift and light transmitted by the beam block does not go through the conjugated aperture (a). In case of high intensities when its phase is shifted by $\lambda / 2$ significant transmission occurs (b).

of the corresponding calculation for a $\lambda / 2$ phase shift. Practically the "inverse" of the annular input beam emerges at the output; as high as $55 \%$ of the input beam is diffracted to the central hole of the output aperture. Another advantage of this method - beyond the high contrast improvement and high overall throughput - that spatial filtering occurs in the central part of the beam, too. According to numerical calculations and experimental observations, the eventual modulation of the input beam is only present in the low intensity, ring-shaped part of the output, which is blocked by output diaphragm.

Self-focusing in a self-generated laser plasma in a gas jet is capable of shifting the phase of the central lobe of the diffraction-pattern by $\lambda / 2$. Experimental realization of the system is shown in Fig. 3a) and 3b) indicating the propagation of a beam of low and high intensity, respectively. Using a pulsed gas jet as a nonlinear phase shifter, constructive interference after passing a nonlinear medium of well defined length (and of no absorption) allows minimization of the losses, and the suppression of the unwanted spatial and temporal components of the laser beam at the same time. The method is self-aligning.

\section{EXPERIMENT}

In our experimental realization a Kepler telescope formed by two lenses of $670 \mathrm{~mm}$ focal length was used for Fourier forward- and back-transformation. A pulsed gas jet of $1 \mathrm{~mm}$ diameter, driven by a typically $1 \mathrm{~ms}$ long pulse generated the nonlinear interaction medium. Argon was selected as the active noble gas. By changing its pressure and the opening time of the valve, the phase shift could be adjusted. At optimum condition when the $\lambda / 2$ phase shift was introduced the inverted output distribution could be demonstrated, as seen from the comparison of Fig. 4a) and 4b). In this arrangement without the detailed optimization of the parameters transmission of $40 \%$ is obtained through the conjugated aperture. 


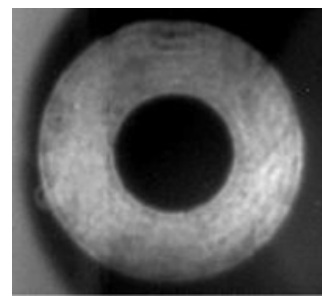

a)

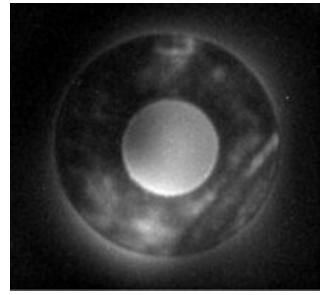

b)

Figure 4. Experimental result for Ar gas jet when no plasma is present (a) and when the generated plasma results in a phase shift of $\sim \lambda / 2(\mathrm{~b})$.

Optimization of the experimental parameters together with theoretical considerations and corresponding measurements for the improvement of the contrast and beam homogeneity are in progress.

\section{SUMMARY}

High contrast temporal and spatial filtering of high-power laser pulses is presented, using a simple, selfadjusting experimental arrangement. The nonlinear plasma filter has high temporal contrast referred to the noise, sharpening the leading edge of the pulse. It has a high transmission ( $>40 \%$ obtained experimentally). It is a beam smoothing tool besides being a spatial filter and it is applicable at any wavelength.

This work was supported by "TÁMOP-4.2.1/B-09/1/KONV-2010-0005 - Creating the Center of Excellence at the University of Szeged" (supported by the European Union and co-financed by the European Regional Development Fund) and it is a part of the COST activity MP0601.

\section{References}

[1] S. Szatmári, G. Marowsky, P. Simon, Femtosecond Excimer Lasers and their Applications, Landolt-Börnstein New Series VIII/1B1, 215 (2007)

[2] S. Szatmári and F.P. Schäfer, Opt. Commun. 68, 196 (1988)

[3] S. Szatmári, Z. Bakonyi, P. Simon, Opt. Commun. 134, 199 (1997)

[4] K.B. Wharton, C.D. Boley, A.M. Komashko, A.M. Rubenchik, J. Zweiback, J. Crane, G. Hays, T.E. Cowan, T. Ditmire; T. Phys. Rev. E, 64, 025401-1-4 (2001)

[5] I.B. Földes, J.S. Bakos, K. Gál, Z. Juhász, M.Á. Kedves, G. Kocsis, S. Szatmári, G. Veres; Laser Phys. 10, 264 (2000)

[6] H. Kapteyn, M. Murnane, A. Szoke, R. Falcone, Opt. Lett. 16, 490 (1991)

[7] I.B. Földes, D. Csáti, F.L. Szúcs, S. Szatmári, Rad. Effects \& Defects in Solids 165, 429 (2010) 